\title{
Universal covariant quantum cloning circuits for qubit entanglement manipulation
}

\author{
Levente Szabó \\ Institute of Physics, University of Pécs, H-7624 Pécs, Ifjúság útja 6, Hungary \\ Mátyás Koniorczyk \\ Institute of Physics, University of Pécs, H-7624 Pécs, Ifjúság útja 6, Hungary and \\ Research group for high performance computing, SP! Project Office, \\ University of Pécs, H-7633 Pécs, Szántó Kovács János u. 1/B. \\ Peter Adam and József Janszky \\ Institute of Physics, University of Pécs, H-7624 Pécs, Ifjúság útja 6, Hungary and \\ Research Institute for Solid State Physics and Optics, \\ Hungarian Academy of Sciences, H-1525 Budapest, P.O. Box 49., Hungary
}

(Dated: November 20, 2009.)

\begin{abstract}
We consider the entanglement manipulation capabilities of the universal covariant quantum cloner or quantum processor circuit for quantum bits. We investigate its use for cloning a member of a bipartite or a genuine tripartite entangled state of quantum bits. We find that for bipartite pure entangled states a nontrivial behavior of concurrence appears, while for GHZ entangled states a possibility of the partial extraction of bipartite entanglement can be achieved.

PACS numbers: 03.67.-a,03.67.Bg
\end{abstract}

\section{INTRODUCTION}

As it is prevalently known, quantum mechanics forbids the copying of individual quantum systems, however, it is still possible to create imperfect replicas of a quantum state with optimal fidelity [1]. This protocol, originally introduced by Bužek and Hillery [2], is called quantum cloning, and it has been studied very extensively (see Ref. 3] for a review).

In this paper we are interested in universal cloning transformations for individual quantum bits. A quantum circuit was designed by Bužek et al. for this purpose [4], which was later generalized to arbitrary dimensional quantum systems [5]. We shall call this circuit UCQC (universal covariant quantum cloner) in what follows. It has a special feature of being quantum controlled, that is, the fidelity ratio of the two clones is controlled by the initial quantum state of two ancillary quantum bits (one of which will carry the clone after the process). This idea turned out to be related to the concept of programmable quantum networks or quantum processors [6]. These are fixed quantum networks which are capable of performing operations on quantum systems in a way that the operation itself is encoded into the initial quantum state of ancillae. It was found that the very circuit for universal quantum cloning is in fact a probabilistic universal quantum processor [7].

In this paper we consider UCQC-s as entanglement manipulation devices. In the context of cloning, one may ask several questions. One may consider the cloning of an entangled quantum state as a whole, in order to obtain similar entangled pairs. For two qubits this has been analyzed in detail by by several authors [8, 9, 10]. In particular, Bužek at al. [11] compare the fidelity of cloning of an entangled pair by global and local operations.
Another approach might be the broadcasting of entanglement, proposed by Bužek et al. [11]. In this case two parties share an entangled pair and use cloners locally to obtain two partially entangled pairs. This protocol attracted a relevant attention in the literature, too. Topics such as state-dependent broadcasting [12], broadcasting of multipartite entangled states: W-states [12, 13], GHZstates [14], and linear optical realizations [15] were discussed in detail. Our present study is motivated mainly by these works.

Consider an entangled pair. It is always interesting to ask what happens to the entanglement if any of the members of the pair is subjected to some quantum information processing protocol. In the case of quantum teleportation, for instance, rather strikingly the teleported qubit inherits the entanglement of the original qubit with its pair. It is rather natural to ask what happens in the case of a universal quantum cloner. The answer for qubit pairs is partly given by Bandyopadhyay and Kar [16]. They show that if a member (or both members) of a maximally entangled qubit pair is subjected to an optimal universal quantum cloning operation, the resulting state is a Werner state. It is likely, however, that a cloning transformation is realized by some quantum circuit, which uses ancillae for carrying out the operation. It is obviously interesting how the entanglement between the different quantum bits of such a scenario (including also ancillae) behaves. In this paper we consider the UCQC as a circuit, not only the cloning operation itself. We calculate entanglement as measured by concurrence. It turns out that the ancillae play a very specific role and the behavior of concurrence shows a rather interesting pattern. The recent optical realization of certain programmable quantum gate arrays [17] also contributes to the relevance of this question. 


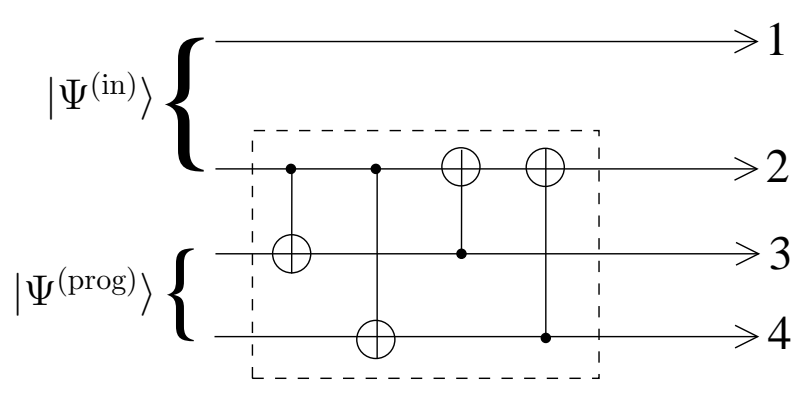

FIG. 1: The setup for bipartite entangled states. The dashed box contains the universal covariant cloning circuit, composed of four controlled-NOT gates.

Another similar question might be the partial extraction of bipartite entanglement from a GHZ-type threepartite resource. It is known that if a three qubits are in a GHZ state [18], then a measurement on either of the three qubits in the $| \pm\rangle$ basis (eigenbasis of the $\sigma_{x}$ Pauli-operator) projects the state of the remaining two qubits into a maximally entangled state. We show that if the given particle is cloned in advance, it is possible to create bipartite entanglement by measuring the clone, while there still remains some purely threepartite entangled resource in the state of the three parties. This is indicated by the possibility of entangling a different pair of qubits by a next measurement. The nature of the entanglement in the multipartite system can be also analyzed with the aid of the Coffman-Kundu-Wootters inequalities [19], which quantify the monogamy of entanglement. We shall present such an analysis, too.

This paper is organized as follows: in Section Iwe analyze the behavior of bipartite entanglement in the case when UCQC is applied to clone a member of a maximally entangled pair. In Section [III we consider the application of UCQC for the partial extraction of bipartite entanglement from a Greenberger-Horne-Zeilinger state. In Section IV the results are summarized and conclusions are drawn.

\section{BIPARTITE PURE STATES}

The considered setup is depicted in Fig. 1. The quantum circuit in the dashed box is the universal quantum cloner [5]. Its first input port (which is port 2 in our current notation) receives the state to be cloned, while on the second two ports there impinges a so-called program state:

$$
\left.\left|\Psi_{34}^{(\mathrm{prog})}\right\rangle=\mathcal{N}(\alpha(|0\rangle(|0\rangle+|1\rangle))+\beta(|00\rangle+|11\rangle))\right)
$$

where $\mathcal{N}=1 / \sqrt{2\left(\alpha+\beta^{2}\right)}$ is a normalization constant and $\beta=1-\alpha$. Were a single-qubit state $\varrho$ impingent on port 2, the output states would be:

$$
\begin{gathered}
\varrho_{2}=\frac{\beta}{\alpha+\beta^{2}} \varrho+\frac{\alpha^{2}}{2\left(\alpha+\beta^{2}\right)} \hat{1}, \\
\varrho_{3}=\frac{\alpha}{\beta+\alpha^{2}} \varrho+\frac{\beta^{2}}{2\left(\beta+\alpha^{2}\right)} \hat{1}, \\
\varrho_{4}=\frac{\alpha \beta}{\beta^{2}+\alpha} \varrho^{T}+\frac{\alpha^{2}+\beta^{2}}{2\left(\alpha+\beta^{2}\right)} \hat{1} .
\end{gathered}
$$

The clones reside in ports 2 and 3 , the original qubit and the first ancilla, whereas in the port 4 there is an ancilla, the state of which is a mixture of the state described by the mixture of the transpose of the density operator of the original state and the identity operator. The fidelity of the clones depends on the value of $\alpha$ : for $\alpha=0$ there is no cloning, whereas for $\alpha=1$ the state of the original qubit is fully transferred to the clone, leaving the original qubit in a completely mixed state. For other values of alpha there are optimal clones generated. Note the symmetry of the formulae in $\alpha$ and $\beta$.

Let us return to the description of the whole scenario in argument, depicted in Fig. 1. The qubits 1 and 2 carry the initial bipartite input state. Qubit 2 is subject to cloning, while qubit 1, the first part of the pair is not manipulated. We are interested in the entanglement relations between the different pairs of qubits in the resulting state. As for the measure of bipartite entanglement for qubits, we apply concurrence according to the Wootters formula: for a quantum state $\varrho$ of two qubits,

$$
\begin{array}{r}
C(\varrho)=\max \left(0, \lambda_{1}-\lambda_{2}-\lambda_{3}-\lambda_{4}\right), \\
\lambda_{i}=\operatorname{eig}_{i}(\sqrt{\sqrt{\varrho} \varrho \sqrt{\varrho}}),
\end{array}
$$

where $\tilde{\varrho}=\sigma_{y} \otimes \sigma_{y} \varrho^{*} \sigma_{y} \otimes \sigma_{y}$ is the Wootters-tilde, $\sigma_{y}$ is the second Pauli-operator, and $\varrho^{*}$ is the transpose of the density matrix $\varrho$ in the product basis [20].

As an input state we consider a state in either of the following four forms:

$$
\begin{array}{r}
\left|\Phi_{12}^{(\mathrm{in})}\right\rangle=\sqrt{C_{0}}|00\rangle \pm \sqrt{C_{1}}|11\rangle, \\
\left|\Psi_{12}^{(\mathrm{in})}\right\rangle=\sqrt{C_{0}}|01\rangle \pm \sqrt{C_{1}}|10\rangle \\
C_{0}+C_{1}=1 .
\end{array}
$$

As for the nonzero concurrences between the various pairs of qubits, we obtain the behavior in Fig. 2, regardless of the choice from the above states. The output states, however, depend on this actual choice, we shall comment on this later.

In the figure one can observe that the entanglement between qubits 1 (the one not manipulated) and qubit 2 (the original qubit) behaves in the similar way as that between qubit 1 and 3 (the one not manipulated and the clone). For $\alpha=0$ (no cloning), qubits 1 and 2 are entangled as they were originally, while for $\alpha=1$, complete cloning, the entanglement is transferred to qubits 1 and 3 , the clone plays the role of the former original qubit 
a) 1-2 (lower surface) and 3-4 (upper surface)

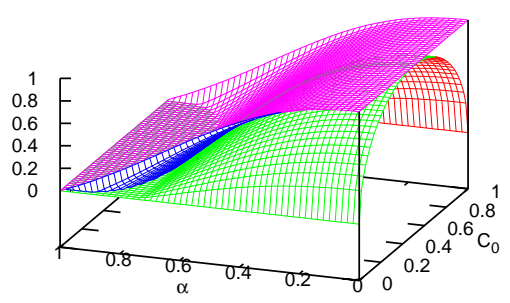

b) $1-3$

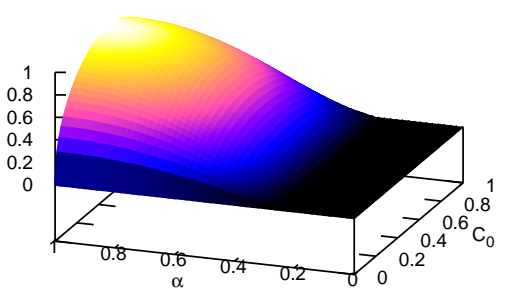

d) $2-3$

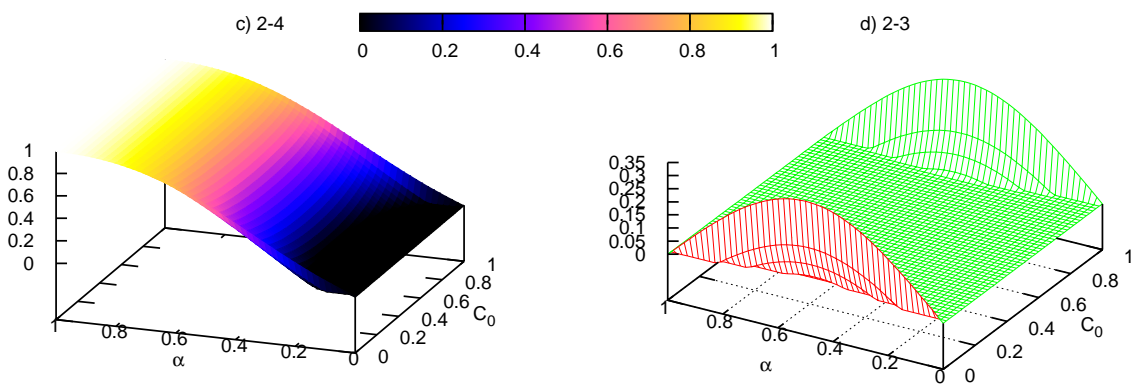

FIG. 2: (color online) The entanglement between the various pairs of qubits at the output of the setup in Fig. 1 The input state is either of the four in Eq. (4), the same figure is obtained for each choice, though the states themselves differ. The "program" state is the one in Eq.(1). The concurrence between qubits 1 and 4 is zero.

completely. The surfaces representing the concurrence for the pairs 1-2 and 1-3 are symmetric in the cloning parameter $\alpha$, that is, they can be obtained from each other by the $\alpha \rightarrow 1-\alpha$ substitution. The dependence of these entanglements from $\alpha$ is monotonous but not continuous: for small values there is a region where the entanglement is zero, and it appears suddenly and non continuously. The dependence of these concurrences on the initial entanglement in the state $C_{0}$ is monotonous and continuous.

It is also interesting to observe that a similar nonsymmetric behavior appears in the concurrence of qubits 3-4 and 2-4. The program state of Eq. (1), in which the qubits 3-4 are prepared initially, is maximally entangled for $\alpha=0$, the case of no cloning, and its entanglement decreases with the increase of the cloning parameter $\alpha$. Accordingly, the entanglement of qubits 3-4 decreases with $\alpha$ also after the cloning operation, while the complementary behavior (in the sense of $\alpha \rightarrow 1-\alpha$ substitution) appears between qubits 2 and 4 (the cloned part of the input state and the ancilla of the cloner). Note that the entanglement of qubits 3 and 4 is not equal to their entanglement before the cloning operation: the concurrence of the partially entangled program state in Eq. (1) is a monotonous and continuous function of $\alpha$, and its values are not equal to the concurrences after the cloning operation. Moreover, the concurrence of qubits 3 and 4 after the cloning also depends slightly on that of the input state of qubits 1 and 2, in Eq. 4.

As for the remaining pairs, qubits 1 and 4 (the qubit not manipulated and the ancilla) will not be entangled, while between qubits 2 and 3 (the second input state and its clone), as a nontrivial effect, there is a small amount of entanglement appearing only in the case the input state (of qubits 1-2) is only slightly entangled.

A special case arises if the input state of qubits 1 and 2 is maximally entangled. This is the case of $C_{0}=1 / 2$ in Fig 2, The concurrence between qubits 1-2 and 34 (two originals, two program qubits of the cloner) is equal to each other. The complementary pairs, qubits 1-3 (not manipulated-clone) and 2-4 (clone-ancilla) have also equal concurrences. The dependence of these concurrences on $\alpha$ is depicted in Fig. 3. The behavior of these curves is due to the fact that the universal cloning transformation produces Werner states. Indeed, if the input state is in Eq. (44) is the maximally entangled $\left|\Phi^{(+)}\right\rangle$ Bell-state, where the states of qubit-pairs 1-2, 1-3, 2-4, 3-4 are Werner-states of the form

$$
\varrho^{(\text {Werner })}=\gamma\left|\Phi^{(+)}\right\rangle\left\langle\Phi^{(+)}\right|+\frac{1-\gamma}{4} \hat{1},
$$

where $\hat{1}$ stands for the identity operator of the two-qubit space. The value of the parameter $\gamma$ is

$$
\gamma_{12}=\gamma_{34}=\frac{\alpha}{\alpha+\beta^{2}}
$$

for pairs 1-2 and 3-4, while it is

$$
\gamma_{13}=\gamma_{24}=\frac{\beta}{\alpha+\beta^{2}} .
$$




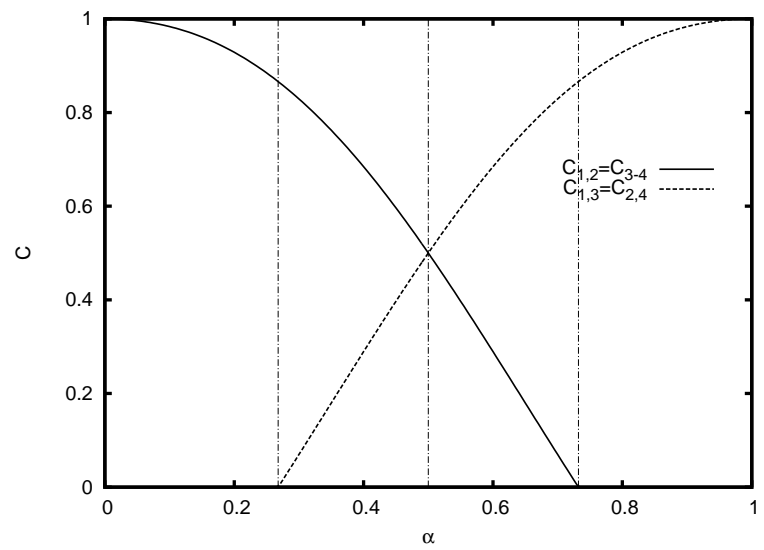

FIG. 3: A slice of Figs. 2 a-c) for $C_{0}=1 / 2$, that is, for any of the maximally entangled Bell-states as input.

Note that the denominator on the right-hand-side of the above formulae comes directly from the normalization constant of the program state in Eq. (11) (i.e. the scaling of parameters in Eqs. (6) and (7) is merely a consequence of our particular choice of parameters). In the case we choose a different one from the states in Eq. (4), we obtain local unitary transforms of the Werner state in Eq. (6). The message of the consideration for cloning an element of a maximally entangled pair is not the fact that Werner states are obtained in qubits 1-2 and 1-3, since it was known from the literature [16]. What is nontrivial here that in the UCQC circuit this behavior is repeated between the ancilla (qubit 4), and qubits 2 and 3, and this holds only in the case of the cloning of a member of a maximally entangled state. Finally let us note that the behavior of qubits 2,3 , and 4 cannot depend on the properties of qubit 1 since it is a remote system from the UCQC's point of view. It is the reduced density operator of qubit 2 which can influence their behavior. We have found that only for a maximally entangled pair, a concurrence characterizing a nonlocal property is equal to another concurrence which is a local property of the cloner.

\section{THE GHZ STATE}

In this section we consider the case in which a member of a Greenberger-Horne-Zeilinger (GHZ) state is cloned. This tripartite state, of the form

$$
\left|\Psi^{(G H Z)}\right\rangle=\frac{1}{\sqrt{2}}(|000\rangle+|111\rangle)
$$

is known to be genuinely tripartite entangled. That is, all the pairwise entanglements (as measured by concurrence) are zero, however, all of the three qubits are in a maximally entangled state. When any of the qubits is

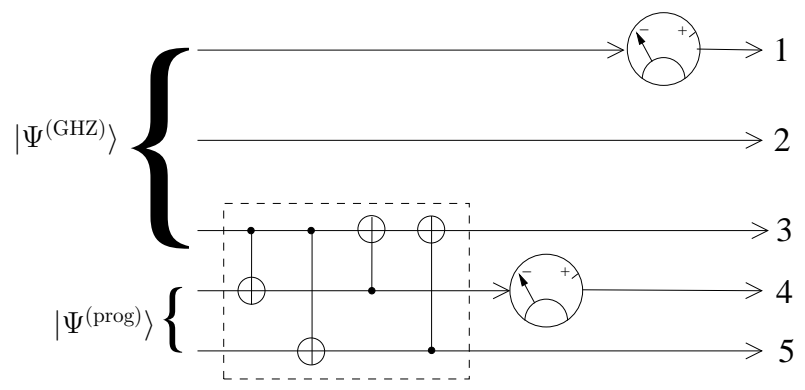

FIG. 4: The setup for the tripartite GHZ state. Qubits 1-3 hold the GHZ state initially. The dashed box contains the universal covariant cloning circuit, composed of four controlledNOT gates. The "meters" measure in the $| \pm\rangle$ basis. First the clone (qubit 3), then a member of the original GHZ state (qubit 1) is measured. The horizontal position from the left to the right side thus represents the order of operations in time.

subject to a von Neumann measurement in the basis

$$
| \pm\rangle=\frac{1}{\sqrt{2}}(|0\rangle \pm|1\rangle)
$$

the other two qubits will be in either of the maximally entangled Bell-states

$$
\left|\Phi^{ \pm}\right\rangle=\frac{1}{\sqrt{2}}(|00\rangle \pm|11\rangle),
$$

depending on the measurement result. The probability of the measurement results are equal. In this way the tripartite entangled resource in the GHZ state can be converted into maximal bipartite entanglement.

The scenario we consider for GHZ states is depicted in Fig. 4. Qubits 1-3 carry the input state which is a GHZ state in Eq. (8). Qubit 3 enters the UCQC's first port. The program ports of the UCQC are qubits 4 and 5 , considered again to be in the program state in Eq. (11).

Directly after the operation of the cloner all pairwise concurrences are zero, except for the one between qubits $3-5$ and $4-5$. Their value is represented by curves "A" and "B" in Fig. 5. This fact is easily explained by the following reasoning. From the point of view of the UCQC circuit, qubits 1 and 2 are remote ones, thus they cannot influence the local properties of qubits 3,4 and 5. All we "see" at the locus of the UCQC is that qubit 3 is in a maximally mixed state, as it is a member of the maximally entangled threepartite GHZ state of Eq (8). But the same situation would arise if qubit 3 were maximally entangled in a bipartite sense with one additional qubit, as we have considered in the previous Section. Thus the behavior of concurrences between the pairs ancillaoriginal and ancilla-clone are the very same as in the case of cloning a member of a bipartite maximally entangled state (or a member of any kind of multipartite entangled state which is itself in a completely mixed state for this reason): Werner states are obtained. 


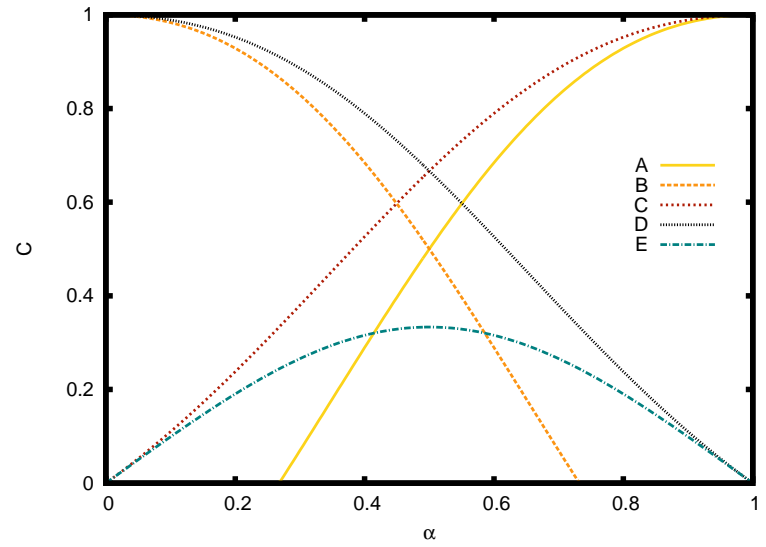

FIG. 5: (color online) Pairwise concurrences in the GHZcloning scenario. A: qubits 3-5 after the cloner and also after each measurement, B: qubits 4-5 after the operation of the cloner, C: qubits 1-2 after the first measurement, D: qubits 23 after the second measurement, E: qubits 2-5 after the second measurement.

a. Projective measurement on the clone. Motivated by the relation of the projective measurements of the members of a GHZ state on the $| \pm\rangle$ basis, one may now consider a measurement of this kind on the clone, that is, on qubit 4 . This measurement will not alter the bipartite entanglement between qubits 3-5, and that between 4-5 will disappear due to the measurement. However, there will be an even larger entanglement appearing between qubits 1 and 2, this is curve "C" in 5, Both measurement outcomes will have equal probability and also the entanglement behavior is the same for both cases. In case of full cloning $(\alpha=1)$, we obtain a pure EPR pair as expected. (We remark here that if we were to measure on the original qubit (qubit 3) instead of its clone, we would obtain the counter propagating curve of the same shape, curve "D" in Fig. 5, as one would expect. The role of the original and the clone is symmetric. Entanglement of 4-5 will not alter, while that of 3-5 will disappear in this case.) This is a partial conversion of the resource available as genuine tripartite entanglement into bipartite entanglement.

In order to further justify this statement let us consider a second measurement, now on qubit 1. Again, the results will be uniformly distributed and the entanglement itself will not depend on the measurement result. The entanglement between qubits 3 and 5 will be untouched, and that between qubits 1 and 2 will be destroyed by the measurement of course. Meanwhile we obtain nonzero entanglement between pairs $2-3$ and $2-5$, these are the curves "D" and "E" in Fig. 5. respectively. Indeed, if the extraction of the tripartite entanglement was not full (i.e. $\alpha \neq 1$, one can still obtain bipartite entanglement by measuring another qubit this time. Curves "C" and "D", describing the entanglement between 1-2 after the first measurement, and 2-3 between the second, respectively, are counter propagating, reflecting the interplay between the two extractions. As a side effect, there is a small amount of entanglement which appears between qubits 2-5 after the second measurement, this is curve "E" in Fig. 囵.

The use of the partial extraction of the entanglement is the following. Consider that qubit 1 is at Alice, qubit 2 at Bob, while the rest of the qubits is at Charlie. Initially they share a tripartite GHZ resource. Charlie wants to enable Alice and Bob to use a bipartite maximally entangled channel. He might perform the projective measurement on the clone he has, however, in this case his qubit 3 gets disentangled from the rest of the parties. However, if he performs cloning and measures the clone, Alice and Bob still obtains a partially entangled bipartite resource. However, Alice can decide that instead of using a bipartite channel with Bob, she wants to create a channel between Bob and Charlie. All she has to do is to perform a proper measurement on her qubit and communicate the result: Bob and Charlie shall posses a partially entangled bipartite resource. This would not be possible without the cloning. The same could be done of course by Bob, to enable the bipartite resource between Alice and Charlie.

In order to obtain a deeper insight into the behavior of bipartite entanglement in this multipartite system, it is worth examining the Coffman-Kundu-Wootters inequalities: for a system of quantum bits in a pure state it always holds that

$$
\tau_{k} \geq \sum_{l \neq k} C_{k, l}^{2}
$$

where $\tau_{k}=4 \operatorname{det} \varrho(k)$ is the one-tangle, the linear entropy of the density operator of the $k$-th qubit. If these inequalities are saturated, the bipartite entanglement is maximal.

To quantify the saturation we evaluate

$$
s=\tau_{k}-\sum_{l \neq k} C_{k, l}^{2}
$$

which is zero if the inequalities are saturated. After the first measurement we obtain nonzero values except for the fourth qubit (apart from the case of $\alpha=1$. The behavior is depicted in Fig. 6. The fact that the CKW inequalities are not saturated also suggests the presence of additional multipartite entanglement in the system. After the second measurement, on the other hand, we find that all the CKW inequalities are saturated: the system is in a sense maximally bipartite entangled.

\section{CONCLUSION}

We have shown that when using a universal covariant quantum cloning circuit to clone a member of an entangled pair of qubits, a very specific behavior of the entanglement of the qubits appears. The main feature is that behavior of the entanglement between the not cloned 


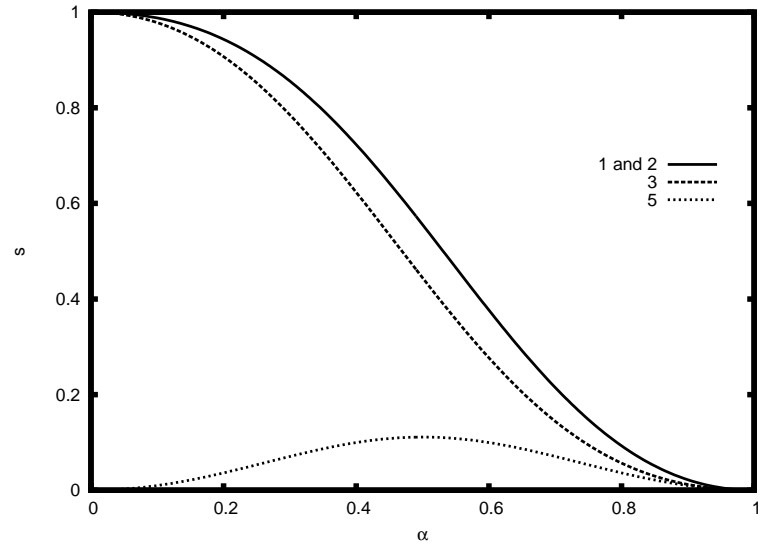

FIG. 6: The quantity in Eq. (12), which is zero if the CKW inequalities are saturated, for each qubit after the first measurement in our GHZ-cloning scenario. For qubit 4 the quantity is zero.

part of the pair and the cloned one is repeated in the entanglement of certain ancillae, and so is that of the not cloned qubit and the clone, provided that the original qubit pair was maximally entangled initially. We have described the behavior of the entanglement in detail.

We have also investigated the cloning of an element of the GHZ state. It appears that the universal quantum cloning circuit facilitates the partial extraction of bipartite entangled resources from a genuine tripartite entangled resource. We provided a detailed analysis of the entanglement behavior, including the relation to CoffmanKundu-Wootters inequalities.

In conclusion, the universal quantum cloning circuit (or quantum processor) for qubits is found to be useful as an entanglement manipulator as well. It can perform entanglement manipulations which are potentially applicable in quantum information processing.

\section{Acknowledgments}

We acknowledge the support of the Hungarian Scientific Research Fund (OTKA) under the contract No. T049234. M.K. acknowledges the support of the project TÁMOP 4.2.2. "SP! IKT - Science, Please! Innovative Research Team".
[1] R. F. Werner, Phys. Rev. A 58, 1827 (1998).

[2] V. Bužek and M. Hillery, Phys. Rev. A 54, 1844 (1996).

[3] V. Scarani, S. Iblisdir, N. Gisin, and A. Acín, Rev. Mod. Phys. 77, 1225 (2005).

[4] V. Bužek, S. L. Braunstein, M. Hillery, and D. Bruß, Phys. Rev. A 56, 3446 (1997).

[5] S. Braunstein, V. Bužek, and M. Hillery, Phys. Rev. A 63, 052313 (2001).

[6] M. A. Nielsen and I. L. Chuang, Phys. Rev. Lett. 79, 321 (1997).

[7] M. Hillery, V. Bužek, and M. Ziman, Phys. Rev. A 65, 022301 (2002).

[8] J. Novotný, G. Alber, and I. Jex, Phys. Rev. A 71, 042332 (2005).

[9] L.-P. Lamoureux, P. Navez, J. Fiurášek, and N. J. Cerf, Phys. Rev. A 69, 040301 (2004).

[10] E. Karpov, P. Navez, and N. J. Cerf, Phys. Rev. A 72, 042314 (2005).

[11] V. Bužek and M. Hillery, Phys. Rev. Lett. 81, 5003
(1998).

[12] S. Adhikari, B. Choudhury, and I. Chakrabarty, J. Phys. A-Math. Gen. 39, 8439 (2006).

[13] I. Chakrabarty and B. S. Choudhury, Int. J. Quantum Inf. 7, 559 (2009).

[14] S. Adhikari and B. S. Choudhury, Phys. Rev. A 74, 032323 (2006).

[15] I. Ghiu and A. Karlsson, Phys. Rev. A 72, 032331 (2005).

[16] S. Bandyopadhyay and G. Kar, Phys. Rev. A 60, 3296 (1999).

[17] M. MiCuda, M. Ježek, M. Dušek, and J. Fiurašek, Phys. Rev. A 78, 062311 (2008).

[18] D. M. Greenberger, M. A. Horne, and A. Zeilinger, Physics Today 46, 22 (1993).

[19] V. Coffman, J. Kundu, and W. K. Wootters, Phys. Rev. A 61, 052306 (2000).

[20] W. K. Wootters, Phys. Rev. Lett. 80, 2245 (1998). 